\title{
PNEUMOPERITÔNIO COM DIÓXIDO DE CARBONO ASSOCIADO A TRÊS POSIÇÕES PARA LAPAROSCOPIA EM CÃES
}

\author{
PNEUMOPERITONEUM USING CARBON DIOXIDE ASSOCIATED WITH THREE \\ POSITIONS FOR LAPAROSCOPY IN DOGS
}

\author{
Marshal Costa Leme ${ }^{1}$ Cláudio Corrêa Natalini ${ }^{2}$ Carlos Afonso de Castro Beck ${ }^{3}$ \\ Maurício Veloso Brun ${ }^{3}$ Emerson Antônio Contesini ${ }^{3}$ \\ Simone Dias de Alves Lima ${ }^{4}$ Rafael Stedile ${ }^{5}$
}

RESUMO

Doze cães foram submetidos ao pneumoperitônio com dióxido de carbono, em pressão constante de $15 \mathrm{mmHg}$, e posicionados em Trendelenburg, Trendelenburg reverso $e$ decúbito horizontal. As variáveis de saturação de oxigênio na hemoglobina, freqüência cardíaca, freqüencia respiratória, pressão arterial média, sistólica e diastólica, o pH, a pressão parcial de $\mathrm{CO}_{2}$ e a pressão parcial de $\mathrm{O}_{2}$ foram mensurados. Somente a freqüência cardíaca, a freqüência respiratória, o $\mathrm{pH}$ e a pressão parcial de $\mathrm{CO}_{2}$ apresentaram diferença estatisticamente significativa em relação ao tempo.

Palavras chave: Trendelenburg, anestesia, cão, dióxido de carbono, monitoração.

\section{SUMMARY}

The present study evaluated the changes in abdominal insufflation with carbon dioxide, with constant pressure of $15 \mathrm{mmHg}$. In this experiment $12 \mathrm{dogs}$, adult mongrels were used. After having installed the pneumoperitonium, the animals were positioned in Trendelenburg, reversed Trendelenburg and horizontal position. In each one of the mentioned positions, the dogs stayed for a period of 30 minutes, for evaluation of alterations in the variables of saturation of oxygen in the hemoglobin, heart rate, breathing rate, arterial blood pressure, $\mathrm{pH}$, partial pressure of $\mathrm{CO}_{2}$ and partial pressure of $\mathrm{O}_{2}$. There was no influences of the positioning on the studied variables. The heart rate, breathing rate, $\mathrm{pH}$ and the partial pressure of $\mathrm{CO} 2$ had significant difference when compared at the time controls.

Key words: Trendelenburg, anesthesia, dog, carbon dioxide, monitoring.

\section{INTRODUÇÃO}

A anestesia para o procedimento cirúrgico laparoscópico surgiu na década de 60 em conjunto com a introdução e rápida adoção da técnica laparoscópica para abordagens ginecológicas. Em 1969 Alexander e colaboradores foram os pioneiros em reportar as considerações anestésicas singulares dessa prática. As características especiais desse procedimento incluem a criação do pneumoperitônio pela insuflação da cavidade abdominal com gás e o posicionamento do paciente em Trendelenburg (corpo inclinado com a cabeça para baixo) para facilitar a visão dos órgãos pélvicos (ALLEN, 1996).

No ano de 1860, Friedrich Trendelenburg popularizou o posicionamento que levaria seu nome. Essa posição é utilizada para procedimentos pélvicos (ginecológico, urológico, e alguns outros) e é obtida pela colocação do paciente em cefalodeclive com inclinação que pode variar de $10^{\circ}$ a $30^{\circ}$ (ALLEN,

\footnotetext{
${ }^{1}$ Médico Veterinário, Doutorando, Universidade Federal de Santa Maria (UFSM). Autor para correspondência. marshalcl@zipmail.com.br.

${ }^{2}$ Médico Veterinário, PhD, Professor Adjunto do Departamento de Clínica de Pequenos Animais, UFSM.

${ }^{3}$ Médico Veterinário, Doutorando, UFSM.

${ }^{4}$ Médico Veterinário, Mestrando, UFSM.

${ }^{5}$ Aluno do Curso de Medicina Veterinária, UFSM, Bolsista de Iniciação Científica CNPq. Recebido para publicação em 28.02.01. Aprovado em 04.07.01
} 
1996). Atualmente, variações dessa posição também são utilizadas para cirurgia pélvica (WILCOX \& VANDAM, 1988), para melhorar o fluxo sangüíneo cerebral, terapia para o choque (ZIPPE $\boldsymbol{e t}$ al., 1985), para tratar embolismo venoso, ingurgitar vasos cervicais para colocação de cateter venoso central, para prevenir aspiração de conteúdo no início de emese, e para se obter anestesia epidural alta (WILCOX \& VANDAM, 1988). Essa posição é importante para a laparoscopia porque afasta da cavidade pélvica o intestino e o cólon, o que é necessário para otimizar a visualização de órgãos e minimizar o risco de ocorrer punção durante a introdução do primeiro trocarte, quando esse é colocado sem visualização direta da cavidade. As mudanças fisiológicas ocasionadas pelo posicionamento ocorrem nas funções respiratória e cardíaca, e as alterações principais são a diminuição da capacidade vital respiratória, aumento da capacidade residual funcional, aumento do esforço respiratório, aumento do retorno venoso e aumento do débito cardíaco (ALLEN, 1996). Por outro lado, quando o paciente é posicionado em Trendelenburg reverso, ocorre diminuição do retorno venoso, e conseqüentemente diminuição do débito cardíaco (STEUER, 1998). Tal posição tem sido utilizada para diminuir o sangramento em cirurgias da cabeça e pescoço (OLDHAM, 1973). As possíveis alterações decorrentes do pneumoperitônio são hipoventilação (ALLEN, 1996), devido à diminuição da complacência tóraco pulmonar, acidose respiratória, compressão da veia cava, possibilidade de pneumotórax (SAUBERMANN, 1993), aspiração de conteúdo gástrico, aumento da resistência venosa, aumento de tensão no miocárdio do ventrículo esquerdo (ALLEN, 1996), aumento da pressão arterial sistólica, da frequiência cardíaca, diminuição da resistência vascular periférica, diminuição da pressão das coronárias podendo levar à isquemia cardíaca (STEUER, 1998), aumento do tônus vagal (bradicardia) (ALLEN, 1996) e embolismo gasoso por dióxido de carbono $\left(\mathrm{CO}_{2}\right)$ (SAUBERMANN, 1993; ALLEN, 1996).

Para a realização da laparoscopia cirúrgica, existe a necessidade da criação de um espaço entre a parede abdominal e as vísceras, para permitir a equipe cirúrgica, visualização e manipulação do conteúdo abdominal (COHEN et al., 1997; STEUER, 1998). Embora métodos mecânicos de distensão da cavidade abdominal existam, a insuflação abdominal por gás é prática padrão (STEUER, 1998).

Os agentes insuflantes utilizados para a produção do pneumoperitônio são dióxido de carbono $\left(\mathrm{CO}_{2}\right)$, óxido nitroso $\left(\mathrm{N}_{2} \mathrm{O}\right)$ (COELHO $\boldsymbol{e t}$ al., 1995; REMEDIOS \& FERGUSON, 1996), nitrogênio $\left(\mathrm{N}_{2}\right)$ (GILROY \& ANSON, 1987), oxigênio $\left(\mathrm{O}_{2}\right)$, ar atmosférico (COELHO et al., 1995; REMEDIOS \& FERGUSON, 1996) e o hélio (He) (FITZGERALD $\boldsymbol{e} \boldsymbol{t}$ al., 1992; LEIGHTON $\boldsymbol{e}$ al., 1993). Como características ideais, o gás deve ser barato, não comburente (COELHO et al., 1995; REMEDIOS \& FERGUSON, 1996; STEUER, 1998), incolor e solúvel na água (STEUER, 1998). Se um gás que suporte combustão é escolhido, o uso da diatermia durante a laparoscopia fornece energia suficiente para uma explosão intracavitária (SHARMA et al., 1996; STEUER, 1998). O gás utilizado também deve ser incolor, de maneira a não interferir na visão do cirurgião, e ser altamente solúvel na água, pois o gás é absorvido pelo peritônio, alcançando a circulação sangüínea. A rápida dissolução, por sua vez, previne o embolismo gasoso, embora a administração direta de gás através da agulha de Veress mal posicionada, ou em um vaso rompido, possa sobrepujar a capacidade do corpo em absorver o gás (STEUER, 1998). $\mathrm{O} \mathrm{N}_{2} \mathrm{O}$ não é um gás normalmente utilizado, devido à grande possibilidade de combustão. Existe ainda muita controvérsia sobre o risco real de combustão, mas em pacientes com disfunção respiratória ou arritmias cardíacas, o óxido nitroso permanece uma alternativa (STEUER, 1998). $\mathrm{O} \mathrm{CO}_{2}$ é o agente mais utilizado em laparoscopia (COELHO et al., 1995; REMEDIOS \& FERGUSON, 1996). Seu largo emprego é justificado pela alta solubilidade no sangue, por não ser explosivo (COELHO et al., 1995; LOUGHLIN, 1996; REMEDIOS \& FERGUSON, 1996), suportando procedimentos que envolvam diatermia (COELHO et al., 1995; LOUGHLIN, 1996) por ter baixo custo e ser facilmente obtido (COELHO et al., 1995; REMEDIOS \& FERGUSON, 1996).

$\mathrm{O} \mathrm{CO}_{2}$ pode produzir aumento do volume minuto, alterações na homeostase do $\mathrm{CO}_{2}$, hipertensão (ALLEN, 1996), aumento da pressão intracraniana (O'LEARY \& RUBENSTEIN, 1996), taquicardia, liberação de agentes vasoativos, arritmias devido à alta pressão de $\mathrm{CO}_{2}$ no sangue arterial $\left(\mathrm{PaCO}_{2}\right)$ e embolismo gasoso (ALLEN, 1996). Portanto a laparoscopia padrão realizada com $\mathrm{CO}_{2}$ possui limitações gerais, estando contraindicada em gestantes, em pacientes portadores de trauma perfurante (pelo risco potencial de embolismo por absorção de $\mathrm{CO}_{2}$ através dos vasos rompidos) e em pacientes com doença intracraniana (O'LEARY \& RUBENSTEIN, 1996). WALLACE et al. (1997) estudaram os efeitos da pressão intraabdominal nos valores de 7,5 e $15 \mathrm{mmHg}$ em 40 
humanos submetidos à colecistectomia. Vinte deles foram posicionados em Trendelenburg reverso, com inclinação de $15^{\circ}$, e os outros 20 em decúbito horizontal dorsal. Os autores afirmaram que a freqüência cardíaca não sofreu alterações durante o pneumoperitônio, e que não houve diferença significativa entre os dois grupos. Já a pressão arterial média (PaM) sofreu elevações nos dois grupos. ISHISAKI et al. (1993) utilizaram 21 cães que foram submetidos à ventilação mecânica, com pneumoperitônio produzido com $\mathrm{CO}_{2}$, mantido por 3 horas, avaliando pressões de 8, 12 e 16mmHg. Estipularam o limite de pressão intra-abdominal que pudesse ser tolerado sem causar colapso cardiovascular ou supressão do fluxo sangüíneo para órgãos abdominais, como também a freqüência cardíaca, a pressão arterial média, e a pressão venosa central nos citados valores pressóricos. Os autores afirmam que a freqüência cardíaca, a pressão arterial média e a pressão venosa central se mantiveram constantes durante todas as pressões estudadas, e indicam pressões menores que $12 \mathrm{mmHg}$ como as que possuem influências mínimas na hemodinâmica abdominal.

O objetivo desse estudo foi o de avaliar em cães, as variações decorrentes do pneumoperitônio produzido por dióxido de carbono, em pressão constante de $15 \mathrm{mmHg}$, nas variáveis de saturação de oxigênio na hemoglobina, freqüência e ritmo cardíaco, freqüência respiratória, pressão arterial média, sistólica, e diastólica, gases sangüíneos e equilíbrio ácido-base, quando submetidos ao posicionamento de Trendelenburg, decúbito horizontal e Trendelenburg reverso.

\section{MATERIAL E MÉTODOS}

No presente estudo, foram utilizados 12 cães, adultos, sem raça definida, 7 machos e 5 fêmeas, com peso variando de 12 a $21 \mathrm{~kg}$, provenientes do Biotério Central da Universidade Federal de Santa Maria (UFSM).

Imediatamente após terem sido selecionados no Biotério da UFSM, os cães foram submetidos a exame clínico, que constou de aferição de temperatura corporal interna, por inserção longitudinal de termômetro digital na mucosa retal, avaliação cardíaca e pulmonar através de ausculta com estetoscópio cardiológico, palpação abdominal e avaliação do tempo de repreenchimento capilar.

Os cães foram submetidos à tricotomia ampla, na face ventral do abdômen, do apêndice xifóide ao púbis, $10 \mathrm{~cm}$ lateral à cadeia mamária, e na face medial dos membros pélvicos. Por meio de catéter venoso, foi realizada a venóclise da cefálica de um dos membros torácicos, para a administração de Ringer com lactato de sódio $\left(10 \mathrm{~m} \ell \cdot \mathrm{kg}^{-1} \cdot \mathrm{h}^{-1}\right)$ durante todo o procedimento operatório.
Como indutor anestésico foi utilizada a associação tiletamina/zolazepam na dose de $5 \mathrm{mgkg}^{-1}$ por via intravenosa (IV). Ao apresentarem relaxamento da articulação temporomandibular, os cães foram intubados com traqueotubo do tipo Magill, de diâmetro compatível à traquéia do cão, sendo então conectado ao sistema circula ${ }^{\mathrm{a}}$ e mantidos com halotano em oxigênio a $100 \%$, administrado em circuito semifechado, através de vaporizador calibrado ${ }^{\mathrm{b}}$. Após a conexão do paciente ao sistema inalatório de anestesia, os 10 primeiros minutos foram utilizados para estabilização anestésica, sendo então ajustados o sensor do oxímetro de pulso ${ }^{c}$, o balonete do monitor de pressão arterial $^{\mathrm{d}}$, e os eletrodos do monitor eletrocardiográfico ${ }^{\mathrm{e}}$ (3 eletrodos). $\mathrm{O}$ sensor do oxímetro de pulso foi aplicado à língua dos cães, fornecendo a saturação de oxigênio na hemoglobina no pulso periférico $\left(\mathrm{SpO}_{2}\right)$. Através do monitor cardíaco em derivação II (DII) de Einthoven, foram registradas a presença ou não de arritmias e a frequiência cardíaca (fc). A frequiência respiratória (fr) foi obtida através da contagem dos movimentos do balão reservatório do aparelho de anestesia durante o período de 1 minuto. $\mathrm{O}$ balonete do monitor de pressão arterial $(\mathrm{Pa})$ foi adaptado em um dos membros torácicos sobre a artéria radial para mensuração da pressão arterial média (PaM), pressão arterial sistólica $(\mathrm{PaS})$ e pressão arterial diastólica $(\mathrm{PaD})$. Ao final desses 10 minutos, os parâmetros das variáveis $\mathrm{SpO}_{2}$, fc, fr, $\mathrm{PaM}, \mathrm{PaS}$ e $\mathrm{PaD}$ foram anotados $\mathrm{em}$ fichas individuais devidamente identificadas. Esse primeiro registro foi denominado de valor controle $(\mathrm{C} 0)$, para posterior comparação com os demais valores obtidos.

Para a coleta de sangue, arterial foi utilizada a artéria femoral de um dos membros pélvicos. Depois de localizada por palpação digital, a artéria femoral foi puncionada, utilizando-se uma agulha hipodérmica $13 \times 4$ acoplada a uma seringa de insulina previamente heparinizada. A punção foi realizada em ângulo de $90^{\circ}$ em relação à superfície da face medial da coxa. Após cada coleta, o sítio de punção recebeu pressão digital, evitando assim a formação de hematomas. $\mathrm{O}$ ar residual, quando presente no interior da seringa, foi totalmente removido, e o bisel de cada agulha hipodérmica foi obstruído com um tampão de borracha, com o objetivo de manter a amostra em condições de anaerobiose. Todas as amostras foram devidamente identificadas e estocadas sob refrigeração a $4^{\circ} \mathrm{C}$, por no máximo 3 horas. Depois desse intervalo, foram encaminhadas para o processamento hemogasométrico ${ }^{\mathrm{f}}$. Essa primeira coleta de sangue arterial foi denominada de $\mathrm{A} 0$, sendo realizada, à semelhança de $\mathrm{CO}$, anteriormente à instalação do pneumoperitônio.

Após o período de indução e estabilização anestésica, os cães foram posicionados em decúbito dorsal. Procedeu-se a antissepsia da pele do 
abdômen, com a sequiência de anti-sépticos álcooliodo-álcool. Seguiu-se a colocação dos panos de campo e a apreensão desses por pinças Backhaus. A instalação do pneumoperitônio teve início com a introdução da agulha de Veress ${ }^{\mathrm{g}}$ na linha alba, em ângulo de $90^{\circ} \mathrm{com}$ a parede abdominal, $2 \mathrm{~cm}$ cranial a cicatriz umbilical. Essa manobra foi realizada com o auxílio de duas pinças Backhaus, utilizadas para tração da parede abdominal, incluindo pele e musculatura abdominal, no sentido ventral. Para verificação de seu correto posicionamento, uma seringa descartável contendo solução salina foi acoplada à agulha de Veress. O êmbolo foi tracionado para verificar a ocorrência de punção visceral acidental. Seguiu-se o segundo teste, que consistiu na instilação de solução salina $0,9 \%$ sobre o canhão da agulha de Veress. Quando corretamente posicionada, as gotas foram aspiradas para a cavidade abdominal por pressão negativa. $\mathrm{Na}$ ausência de resistência, foi injetada salina sobre pressão.

A seguir, o manguito do insuflador automático ${ }^{\mathrm{h}}$ de $\mathrm{CO}_{2}{ }^{\mathrm{i}}$ foi conectado à agulha de Veress e pneumoperitônio instituído com o fluxo de $\mathrm{CO}_{2}$ regulado em $2 \mathrm{~L} \cdot \mathrm{min}^{-1}$. Após a instalação do pneumoperitônio, a agulha de Veress foi retirada. No mesmo local de punção, produziu-se uma incisão de pele e subcutâneo sobre a linha alba, para a introdução de um trocarte de $10 \mathrm{~mm}$ de diâmetro. Através desse, foi inserido um endoscópio rígido ${ }^{j} \mathrm{de}$ $10 \mathrm{~mm}$ de diâmetro, com ângulo de visão de $0^{\circ}$, previamente adaptado a microcâmera ${ }^{\mathrm{k}} \mathrm{e}$ a fonte de luz. As imagens obtidas foram processadas e transmitidas para um monitor de vídeo ${ }^{\mathrm{m}}$. Por meio desse sistema, foi realizada a inspeção visual dos órgãos da cavidade abdominal, avaliando-se possíveis punções viscerais ocasionadas pela penetração da agulha de Veress. Através da válvula do trocarte, foi realizada a manutenção do pneumoperitônio até o final do procedimento.

Foi estudado nesse experimento o pneumoperitônio produzido com $\mathrm{CO}_{2}$ em pressão de $15 \mathrm{mmHg}$. Todos os cães permaneceram sob pressão intra-abdominal constante por um período de 1 hora e 30 minutos para o citado valor pressórico.

Os 12 cães foram separados aleatoriamente em 6 grupos de 2 integrantes. Cada grupo foi representado por uma letra $(\mathrm{A}, \mathrm{B}, \mathrm{C}, \mathrm{D}, \mathrm{E}$, $\mathrm{F})$, e se diferenciou dos demais pela seqüência de posições estabelecida, sendo A $(1,2,3), \mathrm{B}(1,3,2$,), $\mathrm{C}(2,1,3), \mathrm{D}(2,3,1)$, E $(3,1,2)$, e $\mathrm{F}(3,2,1)$. No presente estudo, foram avaliadas as posições Trendelenburg, decúbito dorsal horizontal e Trendelenburg reverso. Essas posições foram representadas por números, isto é; 1-Trendelenburg; 2-decúbito dorsal horizontal; 3-Trendelenburg reverso;

Em cada uma das posições, os cães permaneceram por 30 minutos. Para esse experimento, a posição Trendelenburg foi padronizada como sendo o cefalodeclive com inclinação de $30^{\circ}$, e o Trendelenburg reverso o cefaloaclive com inclinação de $30^{\circ}$.

Após cada animal ser adaptado à primeira posição, os parâmetros de $\mathrm{SpO}_{2}$, fc, ritmo cardíaco, fr, $\mathrm{PaM}, \mathrm{PaS}$ e $\mathrm{PaD}$ foram registrados, obedecendo a intervalos de 15 minutos. As coletas de sangue arterial foram realizadas ao final do tempo de permanência em cada posição (5 minutos finais).

Todos os parâmetros coletados foram submetidos a análise estatística, através do teste de análise de variância (ANOVA) para medidas repetidas, e o teste de comparação de médias de Bonferroni, para os valores de $\mathrm{F}$ significativos. $\mathrm{O}$ nível de significância utilizado foi o de $5 \%(\mathrm{P}<0,05)$.

\section{RESULTADOS}

A introdução da agulha de Veress resultou na perfuração do baço de um cão. Não foram registradas alterações de ritmo cardíaco. Os resultados obtidos das variáveis mensuradas estão representados nas tabelas 1 e 2 . As variáveis representadas na tabela 1 não apresentaram diferença significativa entre os posicionamentos e tampouco em relação aos tempos estudados. A

Tabela 1 - Efeitos da insuflação abdominal produzida com $\mathrm{CO}_{2}$ em pressão constante de $15 \mathrm{mmHg}$, nas variáveis de vaporização de halotano $(\mathrm{V} \%)$, pressão arterial média (PaM), pressão arterial sistólica (PaS), pressão arterial diastólica (PaD), saturação de oxigênio na hemoglobina $\left(\mathrm{SpO}_{2}\right)$ e pressão parcial de oxigênio $\left(\mathrm{PaO}_{2}\right)$.

\begin{tabular}{|c|c|c|c|c|c|c|c|c|c|c|c|c|c|}
\hline \multirow[t]{2}{*}{ Posição } & \multirow[t]{2}{*}{ Tempo } & \multicolumn{2}{|c|}{$\mathrm{V} \%$} & \multicolumn{2}{|c|}{$\mathrm{PaM}(\mathrm{mmHg})$} & \multicolumn{2}{|c|}{$\mathrm{PaS}(\mathrm{mmHg})$} & \multicolumn{2}{|c|}{$\mathrm{PaD}(\mathrm{mmHg})$} & \multicolumn{2}{|c|}{$\mathrm{SpO}_{2}(\%)$} & \multicolumn{2}{|c|}{$\mathrm{PaO}_{2}(\mathrm{mmHg})$} \\
\hline & & Média & SEM & Média & SEM & Média & SEM & Média & SEM & Média & SEM & Média & SEM \\
\hline Controle & $\mathrm{C} 0$ & 1,64 & 0,113 & 80,9 & 6,16 & 109,1 & 7,48 & 65,1 & 6,25 & 98,58 & 2,94 & 300,1 & 27,9 \\
\hline Trendel. & $\mathrm{T} 15$ & 1,81 & 0,113 & 89,9 & 6,16 & 110,3 & 7,48 & 77,0 & 6,25 & 98,58 & 2,94 & & \\
\hline Trendel. & T30 & 1,75 & 0,113 & 90,9 & 6,16 & 112,7 & 7,48 & 79,0 & 6,25 & 99,03 & 2,94 & 332,7 & 27,9 \\
\hline Reverso & $\mathrm{R} 15$ & 1,73 & 0,113 & 73,3 & 6,16 & 96,7 & 7,48 & 59,3 & 6,25 & 98,60 & 2,94 & & 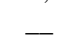 \\
\hline Reverso & R30 & 1,60 & 0,113 & 74,9 & 6,16 & 94,2 & 7,48 & 65,9 & 6,25 & 98,75 & 2,94 & 226,3 & 27,9 \\
\hline Horizont. & H15 & 1,79 & 0,113 & 78,7 & 6,16 & 109,8 & 7,48 & 73,9 & 6,25 & 98,58 & 2,94 & - & 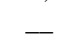 \\
\hline Horizont. & $\mathrm{H} 30$ & 1,66 & 0,113 & 85,8 & 6,16 & 110,3 & 7,48 & 81,3 & 6,25 & 98,91 & 2,94 & $3 \overline{20}, 9$ & $2 \overline{7,9}$ \\
\hline
\end{tabular}

Trendel.=Trendelenburg; reverso=Trendelenburg reverso; horizont.=horizontal; $\mathrm{CO}=$ controle; $\mathrm{SEM=erro} \mathrm{padrão} \mathrm{da} \mathrm{média}(\mathrm{p}<0,05)$.

Ciência Rural, v. 32, n. 2, 2002. 
Tabela 2 - Efeitos da insuflação abdominal produzida com $\mathrm{CO}_{2}$ em pressão constante de $15 \mathrm{mmHg}$, nas variáveis de freqüência respiratória (fr), freqüência cardíaca (fc), percentual de hidrogênio $(\mathrm{pH})$ e pressão parcial de dióxido de carbono $\left(\mathrm{PaCO}_{2}\right)$.

\begin{tabular}{|c|c|c|c|c|c|c|c|c|c|}
\hline \multirow[t]{2}{*}{ Posição } & \multirow[t]{2}{*}{ Tempo } & \multicolumn{2}{|c|}{$\mathrm{fr}(\mathrm{mov} / \mathrm{min})$} & \multicolumn{2}{|c|}{ fc (bat/min) } & \multicolumn{2}{|c|}{$\mathrm{pH}$} & \multicolumn{2}{|c|}{$\mathrm{PaCO}_{2}(\mathrm{mmHg})$} \\
\hline & & Média & SEM & Média & SEM & Média & SEM & Média & SEM \\
\hline Controle & $\mathrm{CO}$ & $21,6^{\mathrm{a}}$ & 5,03 & $138,2^{\mathrm{a}}$ & 7,27 & $7,30^{\mathrm{a}}$ & 0,03 & $47,8^{\mathrm{b}}$ & 7,69 \\
\hline Trendel. & $\mathrm{T} 15$ & $34,8^{\mathrm{b}}$ & 5,03 & $121,1^{\mathrm{b}}$ & 7,27 & & & & \\
\hline Trendel. & T30 & $33,0^{\mathrm{b}}$ & 5,03 & $121,7^{\mathrm{b}}$ & 7,27 & $7 \overline{17^{b}}$ & $\overline{0,03}$ & $7 \overline{4,2}^{\mathrm{b}}$ & $\overline{7,69}$ \\
\hline Reverso & $\mathrm{R} 15$ & $29,5^{\mathrm{b}}$ & 5,03 & $111,3^{\mathrm{b}}$ & 7,27 & & -_ & - & -_ \\
\hline Reverso & $\mathrm{R} 30$ & $29,9^{\mathrm{b}}$ & 5,03 & $104,5^{\mathrm{b}}$ & 7,27 & $7,14^{\mathrm{b}}$ & 0,03 & $71,7^{\mathrm{b}}$ & 7,69 \\
\hline Horizont. & H15 & $31,7^{\mathrm{b}}$ & 5,03 & $116,2^{\mathrm{b}}$ & 7,27 & & - & & \\
\hline Horizont. & $\mathrm{H} 30$ & $30,7^{\mathrm{b}}$ & 5,03 & $115,6^{\mathrm{b}}$ & 7,27 & $7 \overline{19}^{\mathrm{b}}$ & $\overline{0,03}$ & $6 \overline{2,9}^{\mathrm{b}}$ & $\overline{7,69}$ \\
\hline
\end{tabular}

Trendel.=Trendelenburg; reverso=Trendelenburg reverso; horizont.=horizontal; $\mathrm{CO}=$ controle; SEM=erro padrão da média. Letras diferentes indicam diferença significativa pelo Teste de Comparação de Médias de Bonferroni, com nível de significância de 5\%.

tabela 2 representa as variáveis que apresentaram diferença significativa entre os tempos (controle comparado aos valores encontrados aos 15 e aos 30 minutos), mas não entre os posicionamentos.

\section{DISCUSSÃO}

BARATZ \& KARIS (1969), IVANKOVICH $\boldsymbol{e} \boldsymbol{t}$ al. (1975), LEIGHTON $\boldsymbol{e t}$ al. (1993) evidenciaram aumento da $\mathrm{PaCO}_{2}$, com conseqüente diminuição do $\mathrm{pH}$. De maneira similar a esses autores, no presente estudo, em que se fez uso do $\mathrm{CO}_{2}$ como gás insuflante, foram encontrados resultados semelhantes. Tal fato pode ser implicado a absorção de $\mathrm{CO}_{2}$, uma vez que, em estudos comparando os efeitos do pneumoperitônio produzido com $\mathrm{CO}_{2}$ a outros gases, como IVANKOVICH et al. (1975) com o $\mathrm{N}_{2} \mathrm{O}$, e LEIGHTON et al. (1993) com o $\mathrm{He}$, observou-se que eles resultaram em hipercarbia significantemente mais elevada que com $\mathrm{He}$ e o $\mathrm{N}_{2} \mathrm{O}$. Além disso, LEWIS et al. (1972) afirmaram que muitos fatores podem contribuir para o aumento da $\mathrm{PaCO}_{2}$, quando o $\mathrm{CO}_{2}$ é utilizado como gás insuflante, em pacientes anestesiados com halotano e respirando espontaneamente. Dentre esses fatores, destacaram a ação dose dependente de anestésicos, a absorção de $\mathrm{CO}_{2}$ através do peritônio e dificuldade ventilatória por fatores mecânicos, como a distensão abdominal por gás e a posição de Trendelenburg. Embora SCOTT \& SLAWSON (1968) acreditem que a carga extra imposta ao diafragma é superior à suportável pelos músculos respiratórios, a elevação da $\mathrm{PaCO}_{2}$ pode explicar o aumento significativo da frequiência respiratória, na tentativa de restabelecimento do equilíbrio ácido-base. No presente estudo, os autores encontraram hipercapnia devido ao pneumoperitônio com $\mathrm{CO}_{2}$, acarretando acidose respiratória.
SCOTT et al. (1966) citaram que, teoricamente, a posição de Trendelenburg pode afetar a respiração de 3 maneiras. A primeira é o peso visceral sobre o diafragma, que pode dificultar a excursão diafragmática. A segunda, a elevação do diafragma pode diminuir o volume do pulmão. $\mathrm{E}$ por último, a gravidade pode alterar a circulação pulmonar. Contrariamente ao que seria esperado, mas concordando com esses autores, o posicionamento em Trendelenburg não alterou a função respiratória. No presente estudo, a análise dos gases sangüíneos não sofreu alterações devido ao posicionamento; seja Trendelenburg, Trendelenburg reverso ou decúbito horizontal, embora tenha existido diferença estatisticamente significativa $(\mathrm{pH}$ e $\mathrm{PaCO}_{2}$ ), relacionada à duração do pneumoperitônio. Diferente de GROSS et al. (1993), mas de acordo com PURI \& SINGH (1992), a $\mathrm{PaO}_{2}$ não apresentou alterações, assim como a $\mathrm{SpO}_{2}$.

ALLEN (1996) relatou o aumento da fc devido ao pneumoperitônio. SAFRAN \& ORLANDO (1994) observaram aumento da concentração de catecolamina plasmática devido ao pneumoperitônio produzido com $\mathrm{CO}_{2}$. Os autores relacionaram os efeitos cardíacos à liberação desses hormônios, principalmente nos receptores $\beta$. RASSMUSSEN et al. (1978) afirmaram que, devido ao aumento da $\mathrm{PaCO}_{2}$ pela acidose respiratória, ocorre aumento da catecolamina sangüínea. Decorrente da liberação de catecolamina, há o aumento da frequiência cardíaca e da pressão arterial sangüínea. Todavia, os resultados obtidos concordam com TREVOR \& MILLER (1998), que citaram que a depressão cardíaca é um efeito comum devido à utilização do halotano, e STEFFEY (1996) que citou que o efeito dos anestésicos voláteis no músculo cardíaco é dose dependente, causando alterações depressoras, a fc decresceu.

FITZGERALD et al. (1992) utilizaram $\mathrm{CO}_{2}$ e $\mathrm{He}$ para insuflar a cavidade abdominal em pressão de $15 \mathrm{mmHg}$. Observaram elevação significativa da PaM, com os dois gases utilizados para o pneumoperitônio. Concluíram dessa maneira que o aumento da pressão intra-abdominal é o fator preponderante para efeitos observados, e não as propriedades farmacológica ou hormônio estimulantes. No presente estudo, a PaM, diferente de GROSS et al. (1993), ISHIZAKI et al. (1993) e IVANKOVICH et $\boldsymbol{a l}$. (1975) e a PaS, diferente de 
STEUER (1998) não sofreram alterações significativas. A PaD também não foi alterada significativamente.

\section{CONCLUSÕES}

De acordo com os resultados obtidos, e nas condições em que foi realizado este trabalho, pode-se concluir que não existe relação entre os posicionamentos de Trendelenburg, Trendelenburg reverso e decúbito horizontal dorsal com a eficácia na eliminação de $\mathrm{CO}_{2}$ em cães respirando espontaneamente. A elevação da $\mathrm{PaCO}_{2}$ é relacionada à instalação do pneumoperitônio produzido com $\mathrm{CO}_{2}$ que ocasiona acidose respiratória sendo indicada a ventilação mecânica durante o mesmo com $\mathrm{CO}_{2}$. O pneumoperitônio com dióxido de carbono, em pressão constante de $15 \mathrm{mmHg}$ não induz arritmias cardíacas. Os autores sugerem que novos estudos sejam dirigidos, procurando investigar diferentes gases, o consumo de gás utilizado e a relação com a função respiratória durante o pneumoperitônio.

\section{FONTES DE AQUISIÇÃO}

a - Aparelho de Anestesia Inalatória Takaoka KT 13. São Paulo-SP b - Vaporizador Calibrado HB4.2. São Paulo-SP.

c - Oxímetro de Pulso Nellcor. N-200. USA.

d - Monitor de Pressão Arterial Oscilométrico. Critikon. Dinamap 845 XT. USA.

e - Monitor Cardíaco TEB. M-10. São Paulo-SP.

f - Aparelho de Automação Hemogasométrica AVL. Modelo 990. Roswell-GA.

g - Agulha de Veress Edlo. M12. Canoas-RS

$\mathrm{h}$ - Insuflador Eletrônico de $\mathrm{CO}_{2}$ Storz. Eletronic Endoflator. Rio de Janeiro-RJ

i - $\mathrm{CO}_{2}$. White Martins. Canoas-RS.

j - Endoscópio Rígido Storz. Hopkins. Rio de Janeiro-RJ.

k - Microcâmera Storz. Telecam DX NTSC. Rio de Janeiro-RJ.

1 - Fonte de Luz Storz. Xenon Nova. Rio de Janeiro-RJ.

m - Monitor de Vídeo Sony. Trinitron. UK.

\section{REFERÊNCIAS BIBLIOGRAFICAS}

ALLEN, P.D. Anesthesia for minimally invasive surgery. In: LOUGHLIN, K.R., BROOKS, D.C. Principles of endosurgery. Cambridge : Blackwell Science, 1996. Chapter 5, p.54-71

BARATZ, R.A., KARIS, J.H. Blood gas studies during laparoscopy under general anesthesia. Anesthesiology, Philadelphia, v.30, n.4, p.463-464, 1969.

COELHO, J.C.U, MARCHESINI, JB, WIEDERKEHR, J.C. Complicações gerais em videocirurgia. In: COELHO, J.C.U., MARCHESINI, J.B., MALAFAIA, O. Complicações da videocirurgia, da profilaxia ao tratamento. Rio de Janeiro: Medsi, 1995. Cap.4, p.27-47.

COHEN, R.V., SCHIAVON, C.A., MOREIRA FILHO, L., $\boldsymbol{e}$ t al. Pneumoperitônio: técnicas, objetivos e controvérsias. In: COHEN, R.V., MOREIRA FILHO, L., SCHIAVON, C.A
Laparoscopia intervencionista. Conseqüências metabólicas, sistêmicas e imunológicas. 2.ed. Rio de Janeiro : Interlivros, 1997. Cap.3, p.21-24.

FITZGERALD, S.D., ANDRUS, C.H., BAUDENDISTEL, L.J. et al. Hypercarbia during carbon dioxide pneumoperitoneum. Am J Surg, New York, v.163, n.1, p.186-190, 1992.

GILROY, B.A., ANSON, L.W. Fatal air embolism during anesthesia for laparoscopy in a dog. J Am Vet Med Assoc, Chicago, v.190, n.5, p.552-554, 1987.

GROSS, M.E., JONES, B.D., BERGESTRESSER, D.R., et al. Effects of abdominal insufflation with nitrous oxide on cardiorespiratory measumenmentsi in spontaneously breathing isoflurane anesthetized dogs. Am J Vet Res, Chicago, v.5, n.8, p.1352-1358, 1993.

ISHIZAKI, Y., BANDAI, Y., SHIMOMURA, K., et al. Safe intraabdominal pressure of carbon dioxide pneumoperitoneum during laparoscopy surgery. Surgery, Saint Louis, v.114, n.3, p.549-554, 1993.

IVANKOVICH, A.D., MILETICH, D.J., ALBRECHT, R.F., et al. Cardiovascular effects of intraperitoneal insufflations with carbon dioxide and nitrous oxide in the dog. Anesthesiology, Philadelphia, v.42, n.3, p.281-287, 1975.

LEIGHTON, T.A., LIU, S.Y., BONGARD, F.S. Comparative cardiopulmonary effects of carbon dioxide versus helium penumoperitoneum. Surgery, Saint Louis, v.113, n.5, p.527531,1993

LEWIS, D.G., RYDER, W., BURN, N., et al. Laparoscopy-an investigation during spontaneous ventilation with halothane. Br J Anesth, London, v.44, p.685-691, 1972.

LOUGHLIN, K.R. Laparoscopic instrumentation. In: LOUGHLIN, K.R., BROOKS, D.C. Principles of endosurgery. Cambridge : Blackwell Science, 1996. Chapter 1, p.1-8.

O'LEARY, M.P., RUBENSTEIN, S.C. Gassless laparoscopy. In LOUGHLIN, K.R., BROOKS, D.C. Principles of endosurgery. Cambridge : Blackwell Science, 1996. Chapter 6, p.72-80.

OLDHAM, K.W. An unusual complication of the reversed Trendelenburg position. Anaesthesia, Philadelphia, v.28, p.451-452, 1973 .

PURI, G.D., SINGH, H. Ventilatory effects of laparoscopy under general anaesthesia. Br J Anaesth, London, v.68, p.211-213, 1992.

RASMUSSEN, J.P., DAUCHOT, P.J., DePALMA, R.G., $\boldsymbol{e}$ t al Cardiac function and hypercarbia. Arch Surg, Chicago, v.113, p.1196-2000, 1978

REMEDIOS, A.M., FERGUSON, J. Minimally invasive surgery: laparoscopic and thoracoscopy in small animals. Comp Cont Educ Pract Vet, Toronto, v.18, n.11, p.1191-1199, 1996.

SAFRAN, D.B., ORLANDO, R. Physiologic effects of pneumoperitoneum. Am J Surg, New York, v.167, p.281286, 1994.

SAUBERMANN, L.F. Anestesia. In: CREUZ, O. Manual de cirurgia vídeo endoscópica. Rio de Janeiro : Revinter, 1993. Cap.7, p.79-81. 
SCOTT, D.B., LEES, M.M., TAYLOR, S.H. Some respiratory effects of the Trendelenburg position during anaesthesia. $\mathbf{B r}$ J Anaesth, v.38, p.174-184, 1966.

SCOTT, D.B., SLAWSON, K.B. Respiratory effects of prolonged Trendelenburg position. Br J Anaesth, London, v.40, p.103-106, 1968.

SHARMA, K.C., BRANDSTETTER, R.D., BRENSILVER, J.M., et al. Cardiopulmonary physiology and pathophysiology as a consequence of laparoscopic surgery. Chest, Chicago, v.110, n.3, p.810-816, sep 1996.

STEFFEY, E.P. Lumb \& Jones' veterinary anesthesia. 3.ed. Philadelphia : Willians \& Wilkins, 1996. Chap.11: Inhalation anesthetics: p.297-329.

STEUER, K. Pneumoperioneum; phisiology and nursing interventions. AORN J, Denver, v.68, n.3, p.412-425, sept 1998.
TREVOR, A.J., MILLER, R..D. Anestésicos gerais. In : KATZUNG, B.G. Farmacologia básica e clínica. 6.ed. Rio de Janeiro : Guanabara Koogan, 1998. Cap.24, p.291-300.

WALlACE, D.H., SERPELL, M.G., BAXTER, J.N., et al. Randomized trial of different insufflation pressures for laparoscopic cholecystectomy. Br J Surg, Bristol, v.84, n.4, p.455-458, 1997.

WILCOX, S., VANDAM, L.D. Alas poor Trendelenburg and his position! A critique of its uses and effectiveness. Anesth Analg, New York, v.67, p.574-578, 1988.

ZIPPE, C., KENNETH, W.B., GANN, D.S. Trendelenburg versus PASG aplication in moderate hemorragic hypoperfusion. $\mathbf{J}$ Trauma, Baltimore, v.25, n.10, p.923-931, 1985.

Ciência Rural, v. 32, n. 2, 2002. 